ANNALES

POLONICI MATHEMATICI

$89.3(2006)$

\title{
Existence and uniqueness of periodic solutions for a kind of Duffing equation with two deviating arguments
}

\author{
by BINGWEN LiU (Jiaxing)
}

Abstract. We use the coincidence degree to establish new results on the existence and uniqueness of $T$-periodic solutions for a kind of Duffing equation with two deviating arguments of the form

$$
x^{\prime \prime}+C x^{\prime}(t)+g_{1}\left(t, x\left(t-\tau_{1}(t)\right)\right)+g_{2}\left(t, x\left(t-\tau_{2}(t)\right)\right)=p(t) .
$$

1. Introduction. Consider the Duffing equation with two deviating arguments of the form

$$
x^{\prime \prime}+C x^{\prime}(t)+g_{1}\left(t, x\left(t-\tau_{1}(t)\right)\right)+g_{2}\left(t, x\left(t-\tau_{2}(t)\right)\right)=p(t),
$$

where $\tau_{1}, \tau_{2}, p: \mathbb{R} \rightarrow \mathbb{R}$ and $g_{1}, g_{2}: \mathbb{R} \times \mathbb{R} \rightarrow \mathbb{R}$ are continuous functions, $C$ is a constant, $\tau_{1}, \tau_{2}$ and $p$ are $T$-periodic, $g_{1}$ and $g_{2}$ are $T$-periodic in the first argument, and $T>0$. In recent years, the problem of the existence of periodic solutions of (1.1) has been extensively studied. We refer the reader to $[2,4-9]$ and the references cited therein. However, to the best of our knowledge, there exist no results on uniqueness of periodic solutions of (1.1).

The main purpose of this paper is to establish sufficient conditions for the existence and uniqueness of $T$-periodic solutions of (1.1). The results of this paper are new and they complement previously known results.

For ease of exposition, throughout this paper we will adopt the following notations:

$$
|x|_{k}=\left(\int_{0}^{T}|x(t)|^{k} d t\right)^{1 / k}, \quad|x|_{\infty}=\max _{t \in[0, T]}|x(t)| .
$$

2000 Mathematics Subject Classification: 34C25, 34D40.

Key words and phrases: Duffing equation, deviating argument, periodic solution, coincidence degree.

This work was supported by the NNSF (10371034) of China. 
Let

$$
X=\left\{x \in C^{1}(\mathbb{R}, \mathbb{R}) \mid x(t+T)=x(t) \text { for all } t \in \mathbb{R}\right\}
$$

and

$$
Y=\{x \in C(\mathbb{R}, \mathbb{R}) \mid x(t+T)=x(t) \text { for all } t \in \mathbb{R}\}
$$

be two Banach spaces with the norms

$$
\|x\|_{X}=\max \left\{|x|_{\infty},\left|x^{\prime}\right|_{\infty}\right\}, \quad\|x\|_{Y}=|x|_{\infty} .
$$

Define a linear operator $L: D(L) \subset X \rightarrow Y$ by setting

$$
D(L)=\left\{x \in X \mid x^{\prime \prime} \in C(\mathbb{R}, \mathbb{R})\right\}
$$

and for $x \in D(L)$,

$$
L x=x^{\prime \prime}
$$

We also define a nonlinear operator $N: X \rightarrow Y$ by setting

$$
N x=-C x^{\prime}(t)-g_{1}\left(t, x\left(t-\tau_{1}(t)\right)\right)-g_{2}\left(t, x\left(t-\tau_{2}(t)\right)\right)+p(t) .
$$

It is easy to see that

$$
\operatorname{Ker} L=\mathbb{R}, \quad \operatorname{Im} L=\left\{x \in Y \mid \int_{0}^{T} x(s) d s=0\right\} .
$$

Thus $L$ is a Fredholm operator with index zero.

Define the continuous projectors $P: X \rightarrow \operatorname{Ker} L$ and $Q: Y \rightarrow Y$ by setting

$$
P x(t)=x(0)=x(T), \quad Q x(t)=\frac{1}{T} \int_{0}^{T} x(s) d s .
$$

Hence, according to [6, 7], we have $\operatorname{Im} P=\operatorname{Ker} L$ and $\operatorname{Ker} Q=\operatorname{Im} L$. Denoting by $L_{P}^{-1}: \operatorname{Im} L \rightarrow D(L) \cap \operatorname{Ker} P$ the inverse of $\left.L\right|_{D(L) \cap \operatorname{Ker} P \text {, we have }}$

$$
L_{P}^{-1} y(t)=-\frac{t}{T} \int_{0}^{T}(t-s) y(s) d s+\int_{0}^{t}(t-s) y(s) d s .
$$

We also assume that the following condition $(H)$ holds.

$(H)$ For $i=1,2$, there exist a constant $\mu_{i}$ and an integer $K_{i}$ such that

$$
\mu_{i}=\sup _{t \in[0, T]}\left|\tau_{i}(t)-K_{i} T\right| \leq T .
$$

2. Preliminary results. In view of (1.2) and (1.3), the operator equation $L x=\lambda N x$ is equivalent to the equation

$$
x^{\prime \prime}+\lambda\left[C x^{\prime}(t)+g_{1}\left(t, x\left(t-\tau_{1}(t)\right)\right)+g_{2}\left(t, x\left(t-\tau_{2}(t)\right)\right)\right]=\lambda p(t),
$$
where $\lambda \in(0,1)$.

For convenience, we recall the continuation theorem of [4]. 
Lemma 2.1. Let $X$ and $Y$ be two Banach spaces. Suppose that $L: D(L)$ $\subset X \rightarrow Y$ is a Fredholm operator with index zero and $N: X \rightarrow Y$ is $L$-compact on $\bar{\Omega}$, where $\Omega$ is an open bounded subset of $X$. Moreover, assume that the following conditions are satisfied:

(1) $L x \neq \lambda N x$ for all $x \in \partial \Omega \cap D(L), \lambda \in(0,1)$;

(2) $N x \notin \operatorname{Im} L$ for all $x \in \partial \Omega \cap \operatorname{Ker} L$;

(3) the Brouwer degree

$$
\operatorname{deg}\{Q N, \Omega \cap \operatorname{Ker} L, 0\} \neq 0 .
$$

Then the equation $L x=N x$ has at least one T-periodic solution on $\bar{\Omega}$.

The following lemmas will be useful to prove our main results in Section 3 .

Lemma 2.2. If $x \in C^{2}(\mathbb{R}, \mathbb{R})$ with $x(t+T)=x(t)$, then

$$
\left|x^{\prime}(t)\right|_{2}^{2} \leq\left(\frac{T}{2 \pi}\right)^{2}\left|x^{\prime \prime}(t)\right|_{2}^{2}
$$

Proof. Lemma 2.2 is known as Wirtinger's inequality; see $[3,8]$ for its proof.

Lemma 2.3. Assume that

$\left(A_{1}\right)$ one of the following conditions holds:

(1) $\left(g_{i}\left(t, u_{1}\right)-g_{i}\left(t, u_{2}\right)\right)\left(u_{1}-u_{2}\right)>0$ for $i=1,2, u_{i} \in \mathbb{R}, t \in \mathbb{R}$ and $u_{1} \neq u_{2}$,

(2) $\left(g_{i}\left(t, u_{1}\right)-g_{i}\left(t, u_{2}\right)\right)\left(u_{1}-u_{2}\right)<0$ for $i=1,2, u_{i} \in \mathbb{R}, t \in \mathbb{R}$ and $u_{1} \neq u_{2}$;

$\left(A_{2}\right)$ there exists a constant $d>0$ such that one of the following conditions holds:

(1) $x\left(g_{1}(t, x)+g_{2}(t, x)-p(t)\right)>0$ for all $t \in \mathbb{R},|x| \geq d$,

(2) $x\left(g_{1}(t, x)+g_{2}(t, x)-p(t)\right)<0$ for all $t \in \mathbb{R},|x| \geq d$.

If $x(t)$ is a T-periodic solution of $(2.1)_{\lambda}$, then

$$
|x|_{\infty} \leq d+\sqrt{T}\left|x^{\prime}\right|_{2} .
$$

Proof. Let $x(t)$ be a $T$-periodic solution of $(2.1)_{\lambda}$. Set

$$
x\left(t_{\max }\right)=\max _{t \in \mathbb{R}} x(t), \quad x\left(t_{\min }\right)=\min _{t \in \mathbb{R}} x(t), \quad t_{\max }, t_{\min } \in \mathbb{R} .
$$

Then

$$
x^{\prime}\left(t_{\text {max }}\right)=0, \quad x^{\prime \prime}\left(t_{\text {max }}\right) \leq 0, \quad x^{\prime}\left(t_{\text {min }}\right)=0, \quad x^{\prime \prime}\left(t_{\text {min }}\right) \geq 0 .
$$


In view of $(2.1)_{\lambda},(2.4)$ implies that

$$
\begin{array}{r}
g_{1}\left(t_{\max }, x\left(t_{\max }-\tau_{1}\left(t_{\max }\right)\right)\right)+g_{2}\left(t_{\max }, x\left(t_{\max }-\tau_{2}\left(t_{\max }\right)\right)\right)-p\left(t_{\max }\right) \\
=-x^{\prime \prime}\left(t_{\max }\right) / \lambda \geq 0,
\end{array}
$$

and

$$
\begin{array}{r}
g_{1}\left(t_{\min }, x\left(t_{\min }-\tau_{1}\left(t_{\min }\right)\right)\right)+g_{2}\left(t_{\min }, x\left(t_{\min }-\tau_{2}\left(t_{\min }\right)\right)\right)-p\left(t_{\min }\right) \\
=-x^{\prime \prime}\left(t_{\min }\right) / \lambda \leq 0 .
\end{array}
$$

Since $g_{1}\left(t, x\left(t-\tau_{1}(t)\right)\right)+g_{2}\left(t, x\left(t-\tau_{2}(t)\right)\right)-p(t)$ is a continuous function on $\mathbb{R}$, it follows from (2.5) and (2.6) that there exists a constant $t_{1} \in \mathbb{R}$ such that

$$
g_{1}\left(t_{1}, x\left(t_{1}-\tau_{1}\left(t_{1}\right)\right)\right)+g_{2}\left(t_{1}, x\left(t_{1}-\tau_{2}\left(t_{1}\right)\right)\right)-p\left(t_{1}\right)=0 .
$$

Now we prove

Claim. If $x(t)$ is a T-periodic solution of $(2.1)_{\lambda}$, then there exists a constant $t_{2} \in \mathbb{R}$ such that

$$
\left|x\left(t_{2}\right)\right| \leq d
$$

Assume, by way of contradiction, that (2.8) does not hold. Then

$$
|x(t)|>d \quad \text { for all } t \in \mathbb{R},
$$

which, together with $\left(A_{2}\right)$ and (2.7), implies that one of the following relations holds:

$$
\begin{aligned}
& x\left(t_{1}-\tau_{1}\left(t_{1}\right)\right)>x\left(t_{1}-\tau_{2}\left(t_{1}\right)\right)>d ; \\
& x\left(t_{1}-\tau_{2}\left(t_{1}\right)\right)>x\left(t_{1}-\tau_{1}\left(t_{1}\right)\right)>d ; \\
& x\left(t_{1}-\tau_{1}\left(t_{1}\right)\right)<x\left(t_{1}-\tau_{2}\left(t_{1}\right)\right)<-d ; \\
& x\left(t_{1}-\tau_{2}\left(t_{1}\right)\right)<x\left(t_{1}-\tau_{1}\left(t_{1}\right)\right)<-d .
\end{aligned}
$$

Suppose that (2.10) holds. In view of $\left(A_{1}\right)$ and $\left(A_{2}\right)$, we shall consider four cases as follows.

Case (i). If $\left(A_{2}\right)(1)$ and $\left(A_{1}\right)(1)$ hold, then (2.10) implies

$$
\begin{aligned}
0 & <g_{1}\left(t_{1}, x\left(t_{1}-\tau_{2}\left(t_{1}\right)\right)\right)+g_{2}\left(t_{1}, x\left(t_{1}-\tau_{2}\left(t_{1}\right)\right)\right)-p\left(t_{1}\right) \\
& <g_{1}\left(t_{1}, x\left(t_{1}-\tau_{1}\left(t_{1}\right)\right)\right)+g_{2}\left(t_{1}, x\left(t_{1}-\tau_{2}\left(t_{1}\right)\right)\right)-p\left(t_{1}\right),
\end{aligned}
$$

which contradicts $(2.7)$.

CASE (ii). If $\left(A_{2}\right)(1)$ and $\left(A_{1}\right)(2)$ hold, then (2.10) implies

$$
\begin{aligned}
0 & <g_{1}\left(t_{1}, x\left(t_{1}-\tau_{1}\left(t_{1}\right)\right)\right)+g_{2}\left(t_{1}, x\left(t_{1}-\tau_{1}\left(t_{1}\right)\right)\right)-p\left(t_{1}\right) \\
& <g_{1}\left(t_{1}, x\left(t_{1}-\tau_{1}\left(t_{1}\right)\right)\right)+g_{2}\left(t_{1}, x\left(t_{1}-\tau_{2}\left(t_{1}\right)\right)\right)-p\left(t_{1}\right),
\end{aligned}
$$

which contradicts (2.7). 
Case (iii). If $\left(A_{2}\right)(2)$ and $\left(A_{1}\right)(1)$ hold, then (2.10) implies

$$
\begin{aligned}
0 & >g_{1}\left(t_{1}, x\left(t_{1}-\tau_{1}\left(t_{1}\right)\right)\right)+g_{2}\left(t_{1}, x\left(t_{1}-\tau_{1}\left(t_{1}\right)\right)\right)-p\left(t_{1}\right) \\
& >g_{1}\left(t_{1}, x\left(t_{1}-\tau_{1}\left(t_{1}\right)\right)\right)+g_{2}\left(t_{1}, x\left(t_{1}-\tau_{2}\left(t_{1}\right)\right)\right)-p\left(t_{1}\right),
\end{aligned}
$$

which contradicts (2.7).

CASE (iv). If $\left(A_{2}\right)(2)$ and $\left(A_{1}\right)(2)$ hold, then (2.10) implies

$$
\begin{aligned}
0 & >g_{1}\left(t_{1}, x\left(t_{1}-\tau_{2}\left(t_{1}\right)\right)\right)+g_{2}\left(t_{1}, x\left(t_{1}-\tau_{2}\left(t_{1}\right)\right)\right)-p\left(t_{1}\right) \\
& >g_{1}\left(t_{1}, x\left(t_{1}-\tau_{1}\left(t_{1}\right)\right)\right)+g_{2}\left(t_{1}, x\left(t_{1}-\tau_{2}\left(t_{1}\right)\right)\right)-p\left(t_{1}\right),
\end{aligned}
$$

which contradicts (2.7).

If $(2.11),(2.12)$, or $(2.13)$ holds, the reasoning is similar. This completes the proof of the Claim.

Let $t_{2}=m T+t_{0}$, where $t_{0} \in[0, T]$ and $m$ is an integer. Then, using the Schwarz inequality and the relation

$$
|x(t)|=\left|x\left(t_{0}\right)+\int_{t_{0}}^{t} x^{\prime}(s) d s\right| \leq d+\int_{0}^{T}\left|x^{\prime}(s)\right| d s, \quad t \in[0, T],
$$

we obtain

$$
|x|_{\infty}=\max _{t \in[0, T]}|x(t)| \leq d+\sqrt{T}\left|x^{\prime}\right|_{2} .
$$

This completes the proof of Lemma 2.3.

Lemma 2.4. Let $(H)$ and $\left(A_{1}\right)$ hold. Assume that one of the following conditions is satisfied:

$\left(A_{3}\right)$ there exist constants $b_{1}$ and $b_{2}$ such that

$$
\begin{aligned}
& 0 \leq b_{1}\left(\sqrt{2} \mu_{1}+T / \pi\right)+b_{2}\left(\sqrt{2} \mu_{2}+T / \pi\right)<\frac{2 \pi}{T}, \\
& \left|g_{i}\left(t, x_{1}\right)-g_{i}\left(t, x_{2}\right)\right| \leq b_{i}\left|x_{1}-x_{2}\right|,
\end{aligned}
$$

for all $t, x_{i} \in \mathbb{R}, i=1,2$;

$\left(A_{4}\right)$ there exist constants $b_{1}$ and $b_{2}$ such that

$$
\begin{aligned}
& 0 \leq b_{1}\left(\sqrt{2} \mu_{1}+T / \pi\right)+b_{2}\left(\sqrt{2} \mu_{2}+T / \pi\right)<|C|, \\
& \left|g_{i}\left(t, x_{1}\right)-g_{i}\left(t, x_{2}\right)\right| \leq b_{i}\left|x_{1}-x_{2}\right|,
\end{aligned}
$$

for all $t, x_{i} \in \mathbb{R}, i=1,2$.

Then (1.1) has at most one T-periodic solution.

Proof. Suppose that $x_{1}(t)$ and $x_{2}(t)$ are two $T$-periodic solutions of (1.1). Set $Z(t)=x_{1}(t)-x_{2}(t)$. Then

$$
\begin{aligned}
Z^{\prime \prime}(t)+C Z^{\prime}(t) & +\left(g_{1}\left(t, x_{1}\left(t-\tau_{1}(t)\right)\right)-g_{1}\left(t, x_{2}\left(t-\tau_{1}(t)\right)\right)\right) \\
& +\left(g_{2}\left(t, x_{1}\left(t-\tau_{2}(t)\right)\right)-g_{2}\left(t, x_{2}\left(t-\tau_{2}(t)\right)\right)\right)=0 .
\end{aligned}
$$


Integrating (2.14) from 0 to $T$, we get

$$
\begin{aligned}
& \int_{0}^{T}\left[\left(g_{1}\left(t, x_{1}\left(t-\tau_{1}(t)\right)\right)-g_{1}\left(t, x_{2}\left(t-\tau_{1}(t)\right)\right)\right)\right. \\
& \left.+\left(g_{2}\left(t, x_{1}\left(t-\tau_{2}(t)\right)\right)-g_{2}\left(t, x_{2}\left(t-\tau_{2}(t)\right)\right)\right)\right] d t=0 .
\end{aligned}
$$

Thus, in view of the integral mean value theorem, there exists a constant $\gamma \in[0, T]$ such that

$$
\begin{aligned}
g_{1}\left(\gamma, x_{1}(\gamma-\right. & \left.\left.\left.\tau_{1}(\gamma)\right)\right)-g_{1}\left(\gamma, x_{2}\left(\gamma-\tau_{1}(\gamma)\right)\right)\right) \\
& \left.+g_{2}\left(\gamma, x_{1}\left(\gamma-\tau_{2}(\gamma)\right)\right)-g_{2}\left(\gamma, x_{2}\left(\gamma-\tau_{2}(\gamma)\right)\right)\right)=0 .
\end{aligned}
$$

From $\left(A_{1}\right),(2.15)$ implies that

$$
\begin{aligned}
& Z\left(\gamma-\tau_{1}(\gamma)\right) Z\left(\gamma-\tau_{2}(\gamma)\right) \\
& \quad=\left(x_{1}\left(\gamma-\tau_{1}(\gamma)\right)-x_{2}\left(\gamma-\tau_{1}(\gamma)\right)\right)\left(x_{1}\left(\gamma-\tau_{2}(\gamma)\right)-x_{2}\left(\gamma-\tau_{2}(\gamma)\right)\right) \leq 0 .
\end{aligned}
$$

Since $Z(t)=x_{1}(t)-x_{2}(t)$ is a continuous function on $\mathbb{R}$, there exists a constant $\xi \in \mathbb{R}$ such that

$$
Z(\xi)=0 .
$$

Let $\xi=n T+\widetilde{\gamma}$, where $\widetilde{\gamma} \in[0, T]$ and $n$ is an integer. Then (2.16) implies that there exists a constant $\widetilde{\gamma} \in[0, T]$ such that

$$
Z(\widetilde{\gamma})=Z(\xi)=0 .
$$

Let $x(t) \in X$. Suppose that there exists a constant $D \geq 0$ such that

$$
\left|x\left(\tau_{0}\right)\right| \leq D, \quad \tau_{0} \in[0, T] .
$$

Then, from Lemma 2.5 in [5], we obtain

$$
|x|_{2} \leq \frac{T}{\pi}\left|x^{\prime}\right|_{2}+\sqrt{T} D .
$$

Let $\mu \in[0, T]$ be a constant, $\bar{\delta} \in C(\mathbb{R}, \mathbb{R})$ be periodic with period $T$, and $\sup _{t \in[0, T]}|\bar{\delta}(t)| \leq \mu$. Then, for any $h \in X$, from Lemma 1 in [6], we get

$$
\int_{0}^{T}|h(s)-h(s-\bar{\delta}(s))|^{2} d s \leq 2 \mu^{2} \int_{0}^{T}\left|h^{\prime}(s)\right|^{2} d s .
$$

Hence, in view of (2.3) and (2.18), we have

$$
|Z|_{\infty} \leq \sqrt{T}\left|Z^{\prime}\right|_{2}, \quad|Z|_{2} \leq \frac{T}{\pi}\left|Z^{\prime}\right|_{2} .
$$

Now we consider two cases as follows. 
CASE (i). If $\left(A_{3}\right)$ holds, multiplying (2.14) by $Z^{\prime \prime}(t)$ and then integrating from 0 to $T$, from $(2.2),(2.19),(2.20)$ and the Schwarz inequality, we have

$$
\begin{aligned}
& \left|Z^{\prime \prime}\right|_{2}^{2}=\int_{0}^{T}\left|Z^{\prime \prime}(t)\right|^{2} d t \\
= & -\int_{0}^{T}\left[\left(g_{1}\left(t, x_{1}\left(t-\tau_{1}(t)\right)\right)-g_{1}\left(t, x_{2}\left(t-\tau_{1}(t)\right)\right)\right)+\left(g_{2}\left(t, x_{1}\left(t-\tau_{2}(t)\right)\right)\right.\right. \\
& \left.\left.-g_{2}\left(t, x_{2}\left(t-\tau_{2}(t)\right)\right)\right)\right] Z^{\prime \prime}(t) d t \\
\leq & \int_{0}^{T}\left[b_{1}\left|Z\left(t-\tau_{1}(t)\right)\right|+b_{2}\left|Z\left(t-\tau_{2}(t)\right)\right|\right]\left|Z^{\prime \prime}(t)\right| d t \\
\leq & \int_{0}^{T}\left[b_{1}\left(\left|Z\left(t-\tau_{1}(t)+K_{1} T\right)-Z(t)\right|+|Z(t)|\right)\right. \\
& \left.+b_{2}\left(\left|Z\left(t-\tau_{2}(t)+K_{2} T\right)-Z(t)\right|+|Z(t)|\right)\right]\left|Z^{\prime \prime}(t)\right| d t \\
\leq & {\left[b_{1}\left(\sqrt{2} \mu_{1}\left|Z^{\prime}\right|_{2}+|Z|_{2}\right)+b_{2}\left(\sqrt{2} \mu_{2}\left|Z^{\prime}\right|_{2}+|Z|_{2}\right)\right]\left|Z^{\prime \prime}\right|_{2} } \\
\leq & {\left[b_{1}\left(\sqrt{2} \mu_{1}+T / \pi\right)+b_{2}\left(\sqrt{2} \mu_{2}+T / \pi\right)\right] \frac{T}{2 \pi}\left|Z^{\prime \prime}\right|_{2}^{2} . }
\end{aligned}
$$

Since $Z(t), Z^{\prime}(t)$ and $Z^{\prime \prime}(t)$ are $T$-periodic and continuous functions, in view of $\left(A_{3}\right),(2.2),(2.20)$ and $(2.21)$, we have

$$
Z(t) \equiv Z^{\prime}(t) \equiv Z^{\prime \prime}(t) \equiv 0 \quad \text { for all } t \in \mathbb{R} .
$$

Thus, $x_{1}(t) \equiv x_{2}(t)$ for all $t \in \mathbb{R}$. Therefore, (1.1) has at most one $T$-periodic solution.

CASE (ii). If $\left(A_{4}\right)$ holds, multiplying (2.14) by $Z^{\prime}(t)$ and then integrating from 0 to $T$, and applying (2.20), we have

$$
\begin{aligned}
|C|\left|Z^{\prime}\right|_{2}^{2}= & \left.\left|\int_{0}^{T} C\right| Z^{\prime}(t)\right|^{2} d t \mid \\
= & \mid-\int_{0}^{T}\left[\left(g_{1}\left(t, x_{1}\left(t-\tau_{1}(t)\right)\right)-g_{1}\left(t, x_{2}\left(t-\tau_{1}(t)\right)\right)\right)\right. \\
& \left.+\left(g_{2}\left(t, x_{1}\left(t-\tau_{2}(t)\right)\right)-g_{2}\left(t, x_{2}\left(t-\tau_{2}(t)\right)\right)\right)\right] Z^{\prime}(t) d t \mid \\
\leq & \int_{0}^{T}\left[b_{1}\left|x_{1}\left(t-\tau_{1}(t)\right)-x_{2}\left(t-\tau_{1}(t)\right)\right|\right. \\
& \left.+b_{2}\left|x_{1}\left(t-\tau_{2}(t)\right)-x_{2}\left(t-\tau_{2}(t)\right)\right|\right]\left|Z^{\prime}(t)\right| d t
\end{aligned}
$$




$$
\begin{aligned}
& \leq\left[b_{1}\left(\sqrt{2} \mu_{1}\left|Z^{\prime}\right|_{2}+|Z|_{2}\right)+b_{2}\left(\sqrt{2} \mu_{2}\left|Z^{\prime}\right|_{2}+|Z|_{2}\right)\right]\left|Z^{\prime}\right|_{2} \\
& \leq\left[b_{1}\left(\sqrt{2} \mu_{1}+T / \pi\right)+b_{2}\left(\sqrt{2} \mu_{2}+T / \pi\right)\right]\left|Z^{\prime}\right|_{2}^{2} .
\end{aligned}
$$

From (2.18) and $\left(A_{4}\right),(2.22)$ implies that

$$
Z(t) \equiv Z^{\prime}(t) \equiv 0 \quad \text { for all } t \in \mathbb{R} .
$$

Hence, $x_{1}(t) \equiv x_{2}(t)$ for all $t \in \mathbb{R}$. Therefore, (1.1) has at most one $T$ periodic solution. The proof of Lemma 2.4 is now complete.

\section{Main results}

TheOREM 3.1. Let $(H),\left(A_{1}\right)(1)$ and $\left(A_{2}\right)(1)$ hold. Assume that either condition $\left(A_{3}\right)$ or $\left(A_{4}\right)$ is satisfied. Then (1.1) has a unique T-periodic solution.

Proof. By Lemma 2.4, together with $\left(A_{3}\right)$ and $\left(A_{4}\right)$, it is easy to see that (1.1) has at most one $T$-periodic solution. Thus, to prove Theorem 3.1, it suffices to show that (1.1) has at least one $T$-periodic solution. To do this, we shall apply Lemma 2.1. Firstly, we claim that the set of all possible $T$-periodic solutions of $(2.1)_{\lambda}$ is bounded. We consider two cases as follows.

CAse $(1):\left(A_{3}\right)$ holds. Let $x(t)$ be a $T$-periodic solution of $(2.1)_{\lambda}$. Multiplying $(2.1)_{\lambda}$ by $x^{\prime \prime}(t)$ and then integrating from 0 to $T$, in view of $(2.2)$, (2.3), (2.18), (2.19), $\left(A_{3}\right)$ and the Schwarz inequality, we have

$$
\begin{aligned}
& \left|x^{\prime \prime}\right|_{2}^{2}=\int_{0}^{T}\left|x^{\prime \prime}(t)\right|^{2} d t \\
& =-\lambda \int_{0}^{T} C x^{\prime}(t) x^{\prime \prime}(t) d t-\lambda \int_{0}^{T} g_{1}\left(t, x\left(t-\tau_{1}(t)\right)\right) x^{\prime \prime}(t) d t \\
& \quad-\lambda \int_{0}^{T} g_{2}\left(t, x\left(t-\tau_{2}(t)\right)\right) x^{\prime \prime}(t) d t+\lambda \int_{0}^{T} p(t) x^{\prime \prime}(t) d t \\
& \leq \int_{0}^{T}\left|g_{1}\left(t, x\left(t-\tau_{1}(t)\right)\right)\right|\left|x^{\prime \prime}(t)\right| d t+\int_{0}^{T}\left|g_{2}\left(t, x\left(t-\tau_{2}(t)\right)\right)\right|\left|x^{\prime \prime}(t)\right| d t \\
& \quad+\int_{0}^{T}|p(t)|\left|x^{\prime \prime}(t)\right| d t \\
& \leq \int_{0}^{T}\left[\left|g_{1}\left(t, x\left(t-\tau_{1}(t)\right)\right)-g_{1}(t, x(t))\right|\right. \\
& \left.\quad+\left|g_{1}(t, x(t))-g_{2}(t, 0)\right|+\left|g_{1}(t, 0)\right|\right]\left|x^{\prime \prime}(t)\right| d t
\end{aligned}
$$




$$
\begin{aligned}
& +\int_{0}^{T}\left[\left|g_{2}\left(t, x\left(t-\tau_{2}(t)\right)\right)-g_{2}(t, x(t))\right|\right. \\
& \left.+\left|g_{2}(t, x(t))-g_{2}(t, 0)\right|+\left|g_{2}(t, 0)\right|\right]\left|x^{\prime \prime}(t)\right| d t+\int_{0}^{T}|p(t)|\left|x^{\prime \prime}(t)\right| d t \\
\leq & b_{1} \int_{0}^{T}\left|x\left(t-\tau_{1}(t)+K_{1} T\right)-x(t)\right|\left|x^{\prime \prime}(t)\right| d t \\
& +b_{1} \int_{0}^{T}|x(t)|\left|x^{\prime \prime}(t)\right| d t+\int_{0}^{T}\left|g_{1}(t, 0)\right|\left|x^{\prime \prime}(t)\right| d t \\
& +b_{2} \int_{0}^{T}\left|x\left(t-\tau_{2}(t)+K_{2} T\right)-x(t)\right|\left|x^{\prime \prime}(t)\right| d t \\
& +b_{2} \int_{0}^{T}|x(t)|\left|x^{\prime \prime}(t)\right| d t+\int_{0}^{T}\left|g_{2}(t, 0)\right|\left|x^{\prime \prime}(t)\right| d t+\int_{0}^{T}|p(t)|\left|x^{\prime \prime}(t)\right| d t \\
\leq & {\left[b_{1}\left(\sqrt{2} \mu_{1}+T / \pi\right)+b_{2}\left(\sqrt{2} \mu_{2}+T / \pi\right)\right] \frac{T}{2 \pi}\left|x^{\prime \prime}\right|_{2}^{2} } \\
& +\left[\left(b_{1}+b_{2}\right) d+\max \left\{\left|g_{1}(t, 0)\right|+\left|g_{2}(t, 0)\right|: 0 \leq t \leq T\right\}+|p|_{\infty}\right] \sqrt{T}\left|x^{\prime \prime}\right|_{2},
\end{aligned}
$$

which, together with $\left(A_{3}\right)$, implies that there exist positive constants $D_{1}$ and $D_{2}$ such that

$$
\left|x^{\prime \prime}\right|_{2}<D_{1}
$$

and

$$
\left|x^{\prime}\right|_{2}<D_{2}, \quad|x|_{\infty}<D_{2} .
$$

Since $x(0)=x(T)$, there exists a constant $\zeta \in[0, T]$ such that

$$
\begin{aligned}
x^{\prime}(\zeta)=0, \quad\left|x^{\prime}(t)\right| & =\left|x^{\prime}(\zeta)+\int_{\zeta}^{t} x^{\prime \prime}(s) d s\right| \\
& \leq \sqrt{T}\left|x^{\prime \prime}\right|_{2}<\sqrt{T} D_{1} \quad \text { for all } t \in[0, T] .
\end{aligned}
$$

CASE (2): $\left(A_{4}\right)$ holds. Let $x(t)$ be a $T$-periodic solution of $(2.1)_{\lambda}$. Multiplying $(2.1)_{\lambda}$ by $x^{\prime}(t)$ and then integrating from 0 to $T$, by $\left(A_{4}\right),(2.3)$, (2.18), (2.19) and the Schwarz inequality, we have

$$
|C|\left|x^{\prime}\right|_{2}^{2}=\left|\int_{0}^{T} C x^{\prime}(t) x^{\prime}(t) d t\right|
$$$$
=\left|-\lambda \int_{0}^{T} g_{1}\left(t, x\left(t-\tau_{1}(t)\right)\right) x^{\prime}(t) d t-\lambda \int_{0}^{T} g_{2}\left(t, x\left(t-\tau_{2}(t)\right)\right) x^{\prime}(t) d t+\lambda \int_{0}^{T} p(t) x^{\prime}(t) d t\right|
$$ 


$$
\begin{aligned}
\leq & \int_{0}^{T}\left[\left|g_{1}\left(t, x\left(t-\tau_{1}(t)\right)\right)-g_{1}(t, x(t))\right|\right. \\
& \left.+\left|g_{1}(t, x(t))-g_{2}(t, 0)\right|+\left|g_{1}(t, 0)\right|\right]\left|x^{\prime}(t)\right| d t \\
& +\int_{0}^{T}\left[\left|g_{2}\left(t, x\left(t-\tau_{2}(t)\right)\right)-g_{2}(t, x(t))\right|\right. \\
& \left.+\left|g_{2}(t, x(t))-g_{2}(t, 0)\right|+\left|g_{2}(t, 0)\right|\right]\left|x^{\prime}(t)\right| d t+\int_{0}^{T}|p(t)|\left|x^{\prime}(t)\right| d t \\
\leq & {\left[b_{1}\left(\sqrt{2} \mu_{1}+T / \pi\right)+b_{2}\left(\sqrt{2} \mu_{2}+T / \pi\right)\right]\left|x^{\prime}\right|_{2}^{2} \quad } \\
& +\left[\left(b_{1}+b_{2}\right) d+\max \left\{\left|g_{1}(t, 0)\right|+\left|g_{2}(t, 0)\right|: 0 \leq t \leq T\right\}+|p|_{\infty}\right] \sqrt{T}\left|x^{\prime}\right|_{2},
\end{aligned}
$$

This implies that there exists a constant $\bar{D}_{2}>0$ such that

$$
\left|x^{\prime}\right|_{2}<\bar{D}_{2}, \quad|x|_{\infty}<\bar{D}_{2}
$$

Therefore, from (3.4), we obtain

$$
\begin{aligned}
& \left|x^{\prime}(t)\right| \leq\left|x^{\prime}(\zeta)+\int_{\zeta}^{t} x^{\prime \prime}(s) d s\right| \leq\left|\int_{\zeta}^{t} x^{\prime \prime}(s) d s\right| \\
= & \left|\int_{\zeta}^{t}\left[-\lambda C x^{\prime}(s)-\lambda g_{1}\left(s, x\left(s-\tau_{1}(s)\right)\right)-\lambda g_{2}\left(s, x\left(s-\tau_{2}(s)\right)\right)+\lambda p(s)\right] d s\right| \\
\leq & |C| \sqrt{T}\left|x^{\prime}\right|_{2}+T\left[\max _{t \in \mathbb{R},|x| \leq \bar{D}_{2}}\left\{\left|g_{1}(t, x)\right|\right\}+\max _{t \in \mathbb{R},|x| \leq \bar{D}_{2}}\left\{\left|g_{2}(t, x)\right|\right\}+|p|_{\infty}\right] \\
\leq & |C| \sqrt{T} \bar{D}_{2}+T\left[\max _{t \in \mathbb{R},|x| \leq \bar{D}_{2}}\left\{\left|g_{1}(t, x)\right|\right\}+\max _{t \in \mathbb{R},|x| \leq \bar{D}_{2}}\left\{\left|g_{2}(t, x)\right|\right\}+|p|_{\infty}\right] \\
:= & \bar{D}_{1} .
\end{aligned}
$$

Therefore, in view of $(3.3),(3.4),(3.6)$ and (3.7), there exists a positive constant $M_{1}>\max \left\{\sqrt{T} D_{1}+D_{2}, \bar{D}_{1}+\bar{D}_{2}\right\}$ such that

$$
\|x\|_{X} \leq|x|_{\infty}+\left|x^{\prime}\right|_{\infty}<M_{1}
$$

If $x \in \Omega_{1}=\{x \in \operatorname{Ker} L \cap X \mid N x \in \operatorname{Im} L\}$, then there exists a constant $M_{2}$ such that

$$
x(t) \equiv M_{2}, \quad \int_{0}^{T}\left[g_{1}\left(t, M_{2}\right)+g_{2}\left(t, M_{2}\right)-p(t)\right] d t=0 .
$$

Thus,

$$
|x(t)| \equiv\left|M_{2}\right|<d \quad \text { for all } x(t) \in \Omega_{1} \text {. }
$$


Let $M=M_{1}+d+1$. Set

$$
\Omega=\left\{\left.x \in X|| x\right|_{\infty}<M,\left|x^{\prime}\right|_{\infty}<M\right\} .
$$

It is easy to see from (1.3) and (1.4) that $N$ is $L$-compact on $\bar{\Omega}$. We deduce from (3.8), (3.9) and the inequality $M>\max \left\{M_{1}, d\right\}$ that conditions (1) and (2) in Lemma 2.1 hold.

Furthermore, define a continuous function $H(x, \mu)$ by setting

$$
H(x, \mu)=-(1-\mu) x-\mu \cdot \frac{1}{T} \int_{0}^{T}\left[g_{1}(t, x)+g_{2}(t, x)-p(t)\right] d t, \quad \mu \in[0,1] .
$$

In view of $\left(A_{2}\right)(1)$, we obtain

$$
x H(x, \mu) \neq 0 \quad \text { for all } x \in \partial \Omega \cap \operatorname{Ker} L .
$$

Hence, using the homotopy invariance theorem, we have

$\operatorname{deg}\{Q N, \Omega \cap \operatorname{Ker} L, 0\}$

$$
\begin{aligned}
& =\operatorname{deg}\left\{-\frac{1}{T} \int_{0}^{T}\left[g_{1}(t, x)+g_{2}(t, x)-p(t)\right] d t, \Omega \cap \operatorname{Ker} L, 0\right\} \\
& =\operatorname{deg}\{-x, \Omega \cap \operatorname{Ker} L, 0\} \neq 0 .
\end{aligned}
$$

In view of all the discussion above, Lemma 2.1 yields the conclusion of Theorem 3.1.

A similar argument leads to

Theorem 3.2. Let $(H),\left(A_{1}\right)(2)$ and $\left(A_{2}\right)(2)$ hold. Assume that either $\left(A_{3}\right)$ or $\left(A_{4}\right)$ is satisfied. Then (1.1) has a unique T-periodic solution.

\section{Examples and remarks}

ExAmple 4.1. Let $g_{i}(t, x)=\frac{10}{3} x$ for all $t \in \mathbb{R}, x>0$, and $g_{i}(t, x)=$ $\arctan x$ for all $t \in \mathbb{R}, x \leq 0, i=1,2$. Then the Duffing equation

$x^{\prime \prime}+210 x^{\prime}+g_{1}\left(t, x\left(t-\frac{1}{100} \sin ^{2} t\right)\right)+g_{2}\left(t, x\left(t-\frac{1}{300} e^{\cos t}\right)\right)=\frac{1}{4} e^{\cos ^{2} t}$ has a unique $2 \pi$-periodic solution.

Proof. By (4.1), we have $b_{1}=b_{2}=\frac{10}{3}, C=210, \tau_{1}(t)=\frac{1}{100} \sin ^{2} t$, $\tau_{2}(t)=\frac{1}{100} e^{\cos t}$ and $p(t)=\frac{1}{4} e^{\cos ^{2} t}$. It is obvious that the assumptions $(H)$, $\left(A_{1}\right)(1),\left(A_{2}\right)(1)$ and $\left(A_{4}\right)$ hold. Hence, by Theorem $3.1,(4.1)$ has a unique $2 \pi$-periodic solution.

Remark 4.1. (4.1) is a very simple version of Duffing equation. Since $C \neq 0, \tau_{1} \neq 0$ and $\tau_{2} \neq 0$, no results in $[2,4-9]$ and the references therein 
are applicable to (4.1) to obtain the existence and uniqueness of $2 \pi$-periodic solutions. This implies that the results of this paper are essentially new.

REMARK 4.3. By using the methods similar to those used for (1.1), we can deal with the Duffing equation with multiple deviating arguments of the following type:

$$
x^{\prime \prime}+C x^{\prime}(t)+\sum_{i=1}^{n} g_{i}\left(t, x\left(t-\tau_{i}(t)\right)\right)=p(t),
$$

where $\tau_{i}, p: \mathbb{R} \rightarrow \mathbb{R}$ and $g_{i}: \mathbb{R} \times \mathbb{R} \rightarrow \mathbb{R}, i=1, \ldots, n$, are continuous functions, $\tau_{i}$ and $p$ are $T$-periodic, $g_{i}$ are $T$-periodic in the first argument, and $T>0$. One may also establish results similar to those in Theorems 3.1 and 3.2 under some minor additional assumptions on $g_{i}(t, x)$.

\section{References}

[1] T. A. Burton, Stability and Periodic Solutions of Ordinary and Functional Differential Equations, Academic Press, Orlando, FL, 1985.

[2] R. E. Gaines and J. Mawhin, Coincidence Degree and Nonlinear Differential Equations, Lecture Notes in Math. 568, Springer, 1977.

[3] G. H. Hardy, J. E. Littlewood and G. Pólya, Inequalities, Cambridge Univ. Press, 1964.

[4] X. K. Huang and Z. G. Xiang, On existence of $2 \pi$-periodic solutions for delay Duffing equation $x^{\prime \prime}+g(t, x(t-\tau(t)))=p(t)$, Chinese Sci. Bull. 39 (1994), 201-203.

[5] B. W. Liu and L. H. Huang, Periodic solutions for a class of forced Liénard-type equations, Acta Math. Appl. Sinica English Ser. 21 (2005), 81-92.

[6] S. P. Lu and W. G. Ge, Periodic solutions for a kind of Liénard equations with deviating arguments, J. Math. Anal. Appl. 249 (2004), 231-243.

[7] -, - , Some new results on the existence of periodic solutions to a kind of Rayleigh equation with a deviating argument, Nonlinear Anal. 56 (2004), 501-514.

[8] J. Mawhin, Periodic solutions of some vector retarded functional differential equations, J. Math. Anal. Appl. 45 (1974), 588-603.

[9] G. Q. Wang, A priori bounds for periodic solutions of a delay Rayleigh equation, Appl. Math. Lett. 12 (1999), 41-44.

College of Mathematics and Information Science

Jiaxing University

Jiaxing, Zhejiang 314001, P.R. China

E-mail: liubw007@yahoo.com.cn 